\title{
On an exactly solvable toy model and its dynamics
}

\author{
Yang $\mathbf{K} \mathbf{L}^{1,2}$ and Zhang $\mathbf{J} \mathbf{M}^{1,2}$ \\ ${ }^{1}$ Fujian Provincial Key Laboratory of Quantum Manipulation and New Energy \\ Materials, College of Physics and Energy, Fujian Normal University, Fuzhou 350007, \\ China \\ ${ }^{2}$ Fujian Provincial Collaborative Innovation Center for Optoelectronic \\ Semiconductors and Efficient Devices, Xiamen 361005, China
}

\begin{abstract}
The eigenstates and eigenenergies of a toy model, which arose in idealizing a local quenched tight-binding model in a previous publication [Zhang and Yang, EPL 114, 60001 (2016)], are solved analytically. This enables us to study its dynamics in a different way. This model can serve as a good exercise in quantum mechanics at the undergraduate level.
\end{abstract}

PACS numbers: 03.65.-W, 03.65.Ge 


\section{Introduction}

Exactly solvable models are rare, and it is especially the case when accessibility to undergraduate students is required. In a typical undergraduate quantum mechanics course, the few exactly solvable models mentioned are often limited to the infinite square well potential, the hydrogen atom, the harmonic oscillator, and a charged particle in a uniform magnetic field. But it is definitely a good idea to have more such models in store and use them as exercises in teaching. From this point of view, it is a shame that the pedagogical value of some simple models in research articles [1, 2, 3, 4, has not yet been fully appreciated.

In this paper, we would like to promote the awareness of a recently devised toy model, which is exactly solvable and can make a good exercise for students. The model was motivated by the study of the quench dynamics of a Bloch state on a tight-binding model [5, 6, 7] and accounts for the peculiarities of the dynamics very well. Here we detach it from the original context and formulate it in general terms. It consists of infinitely many levels $\{|n\rangle, n \in \mathbb{Z}\}$, and its Hamiltonian is $H=H_{0}+H_{1}$, where the diagonal part $H_{0}$ is

$$
H_{0}=\sum_{n=-\infty}^{\infty} n \Delta|n\rangle\langle n|,
$$

which means that the levels are equally spaced with the gap between two adjacent levels being $\Delta$; and the off-diagonal part $H_{1}$ is

$$
H_{1}=\sum_{n_{1}, n_{2}=-\infty}^{\infty} g\left|n_{1}\right\rangle\left\langle n_{2}\right|,
$$

which means that two arbitrary levels, regardless of their energy difference, are coupled to each other with constant strength $g$. For the Hamiltonian to be hermitian, the two parameters $\Delta$ and $g$ of course should be real.

For those who know the Stey-Gibberd model [8, 9], which is important for modeling quantum decay and quantum resonance [10, 11, 12, 13, 14, the present model might look very similar. However, the difference is substantial. The Stey-Gibberd model is of the Hamiltonian

$$
H_{S G}=\sum_{n=-\infty}^{\infty} n \Delta|n\rangle\left\langle n\left|+E_{d}\right| d\right\rangle\langle d|+g \sum_{n=-\infty}^{\infty}(|d\rangle\langle n|+| n\rangle\langle d|) .
$$

It consists of an equidistant quasi-continuum $\{|n\rangle, n \in \mathbb{Z}\}$ plus a defect mode $|d\rangle$, while

here we have only the quasi-continuum. Moreover, in the Stey-Gibberd model, there is no coupling between the levels belonging to the quasi-continuum band, while here any two of them are coupled.

\section{Stationary properties}

While the authors of Refs. [5, 6, 7] studied the dynamics of the model, they did that by solving the time-dependent Schrödinger equation directly without solving the eigenstates 
and eigenenergies of the model. It is our purpose to fill this gap below.

Even without calculation, we can see that if $|\psi\rangle=\sum_{n=-\infty}^{\infty} a_{n}|n\rangle$ is an eigenstate of $H$ with eigenenergy $E$, the translated state $|\tilde{\psi}\rangle=\sum_{n=-\infty}^{\infty} a_{n}|n+1\rangle$ is an eigenstate with energy $E+\Delta$. Formally, we can introduce the translation (raising) operator $T=\sum_{n=-\infty}^{\infty}|n+1\rangle\langle n|$. It is then readily checked that $\left[H_{0}, T\right]=\Delta T$ and $\left[H_{1}, T\right]=0$. Hence

$$
H T|\psi\rangle=\left(T H_{0}+\Delta T+T H_{1}\right)|\psi\rangle=(\Delta T+T H)|\psi\rangle=(E+\Delta) T|\psi\rangle,
$$

which means the state $|\tilde{\psi}\rangle=T|\psi\rangle$ is an eigenstate of $H$ with eigenenergy $E+\Delta$. Therefore, the eigenenergies are equally spaced too.

To determine their precise values, we project the two sides of the equation $H|\psi\rangle=E|\psi\rangle$ onto the basis state $|n\rangle$. We get $E a_{n}=n \Delta a_{n}+g S$, with

$$
S=\sum_{n=-\infty}^{\infty} a_{n}
$$

The observation is that $S$ is independent of $n$. We solve formally

$$
a_{n}=\frac{g S}{E-n \Delta} .
$$

When this expression is substituted back into (5), the factor $S$ drops out, and we get an equation for $E$,

$$
1=\sum_{n=-\infty}^{\infty} \frac{g}{E-n \Delta} .
$$

By the famous formula [15]

$$
\pi \cot \pi x=\sum_{n=-\infty}^{\infty} \frac{1}{x-n}, \quad x \notin \mathbb{Z},
$$

we then reduces (7) to $1=(\pi g / \Delta) \cot (\pi E / \Delta)$, from which we solve finally the eigenenergies as $(-\infty<m<\infty)$

$$
E_{m}=\Delta\left(m+\frac{1}{\pi} \arctan \frac{\pi g}{\Delta}\right) .
$$

Indexed by the integer $m$, they are indeed equally spaced by $\Delta$. It is convenient to introduce the variable

$$
\alpha=\frac{1}{\pi} \arctan \frac{\pi g}{\Delta}
$$

which takes value in the interval $\left(-\frac{1}{2}, \frac{1}{2}\right)$, so that we can write $E_{m}=\Delta(m+\alpha)$.

We have yet to determine the corresponding eigenstate $\left|\psi_{m}\right\rangle$, i.e., the coefficients $a_{n}$. By ([6) $)$, we know $a_{n}=C /\left(E_{m} / \Delta-n\right)=C /(m-n+\alpha)$. The normalization condition requires

$$
1=\sum_{n=-\infty}^{\infty}\left|a_{n}\right|^{2}=|C|^{2} \sum_{n=-\infty}^{\infty} \frac{1}{(m-n+\alpha)^{2}}
$$


By the formula

$$
\left(\frac{\pi}{\sin \pi x}\right)^{2}=\sum_{n=-\infty}^{\infty} \frac{1}{(x-n)^{2}},
$$

which can be obtained from (8) by differentiation with respect to $x$, we solve

$$
|C|^{2}=\frac{(\pi g / \Delta)^{2}}{\pi^{2}\left[1+(\pi g / \Delta)^{2}\right]}=\frac{\sin ^{2} \pi \alpha}{\pi^{2}},
$$

which is independent of the state index $m$. This finishes the solution of the eigenvalues and eigenstates.

\section{A brief digression}

One might wonder what characteristic of the model is responsible for its exact solvability. We would say that it is the rank-one property of the perturbation $H_{1}$. Introducing the state $|\phi\rangle=\sum_{n \in \mathbb{Z}}|n\rangle$, we can write $H_{1}$ succinctly as $H_{1}=g|\phi\rangle\langle\phi|$. It is then clear that the matrix corresponding to $H_{1}$ is of rank one, the lowest rank possible for a nonzero matrix. Noting that in problems with matrices and integral equations, rank-one perturbations [16] and rank-one kernels [17], respectively, often allow simple analysis, here one can also anticipate some simplicity.

Let us consider a more general model. Let $J$ be an arbitrary subset of $\mathbb{Z}$, and let the Hamiltonians be $H_{0}=\sum_{n \in J} \omega_{n}|n\rangle\langle n|$ and $H_{1}=g|\varphi\rangle\langle\varphi|$. Here the energies $\omega_{n}$ of the levels $|n\rangle$ are arbitrary (but real of course) and the state $|\varphi\rangle=\sum_{n \in J} c_{n}|n\rangle$ is also

arbitrary. In comparison with (11) and (2), here we have dropped some specialities of $H_{0,1}$ but have retained the rank-one feature of $H_{1}$.

Consider the eigenvalue equation $\left(H_{0}+H_{1}\right)|\psi\rangle=E|\psi\rangle$. Projecting the two sides onto $|n\rangle$, we get $E\langle n \mid \psi\rangle=\omega_{n}\langle n \mid \psi\rangle+g\langle n \mid \varphi\rangle\langle\varphi \mid \psi\rangle$, from which we solve

$$
\langle n \mid \psi\rangle=\frac{g c_{n}}{E-\omega_{n}}\langle\varphi \mid \psi\rangle .
$$

$\operatorname{But}\langle\varphi \mid \psi\rangle=\sum_{n \in J}\langle\varphi \mid n\rangle\langle n \mid \psi\rangle=\sum_{n \in J} c_{n}^{*}\langle n \mid \psi\rangle$, hence

$$
\langle\varphi \mid \psi\rangle=\sum_{n \in J} \frac{g\left|c_{n}\right|^{2}}{E-\omega_{n}}\langle\varphi \mid \psi\rangle .
$$

Factoring out the unknown $\langle\varphi \mid \psi\rangle$, we get the equation for the eigenvalue $E$,

$$
1=\sum_{n \in J} \frac{g\left|c_{n}\right|^{2}}{E-\omega_{n}}
$$

This equation is apparently simpler, both analytically and numerically, than what one would obtain from the condition $\operatorname{det}\left(E-H_{0}-H_{1}\right)=0$ in case $H_{1}$ lacks the rank-one property. Further simplification occurs as in (7) when $J=\mathbb{Z}, \omega_{n}=n \Delta$, and $c_{n} \equiv 1$.

Finally, we would like to point out that at least in one dimension, a rank-one perturbation can be readily realized with a delta potential. Actually, this is how it is realized in the problem in Refs. [5, 6, 7]. Suppose $H_{0}=-\frac{\partial^{2}}{\partial x^{2}}+V(x)$ is the unperturbed Hamiltonian of a particle in a one-dimensional potential $V(x)$, whose 
eigenstates $\left\{\chi_{n \geq 1}(x)\right\}$ are all normalizable. Now turn on a delta potential at $x_{0}$, i.e., add a potential $H_{1}=g \delta\left(x-x_{0}\right)$ to $V(x)$. In the basis of $\left\{\chi_{n \geq 1}(x)\right\}, H_{0}$ is diagonal, while the matrix elements of $H_{1}$ are

$$
\left\langle\chi_{m}\left|H_{1}\right| \chi_{n}\right\rangle=g \int_{-\infty}^{+\infty} d x \chi_{m}^{*}(x) \delta\left(x-x_{0}\right) \chi_{n}(x)=g \chi_{m}^{*}\left(x_{0}\right) \chi_{n}\left(x_{0}\right),
$$

which apparently correspond to a rank-one matrix.

\section{Dynamics}

Having obtained the eigenstates and eigenvalues explicitly, we can then use them to solve dynamical problems. For example, let us revisit the quench problem in Refs. [5, 6, 7], which was solved in a different approach. Suppose initially the system is in the level $|0\rangle$. As time evolves, due to the coupling $H_{1}$, all other levels will be populated too. A quantity of primary concern is the probability of transition into an arbitrary level $|n\rangle$. That is, we are interested in the matrix element of the time evolution operator $(\hbar=1)$

$$
A_{n}(t)=\left\langle n\left|e^{-i H t}\right| 0\right\rangle,
$$

whose magnitude squared is the transition probability from $|0\rangle$ to $|n\rangle$ (or survival probability when $n=0$ ).

Inserting the identity operator $I=\sum_{m \in \mathbb{Z}}\left|\psi_{m}\right\rangle\left\langle\psi_{m}\right|$, we get

$$
\begin{aligned}
A_{n}(t) & =\sum_{m \in \mathbb{Z}}\left\langle n \mid \psi_{m}\right\rangle\left\langle\psi_{m} \mid 0\right\rangle e^{-i E_{m} t} \\
& =\sum_{m \in \mathbb{Z}} \frac{|C|^{2}}{(m-n+\alpha)(m+\alpha)} e^{-i(m+\alpha) \Delta t} .
\end{aligned}
$$

It is easily seen that

$$
A_{n}(t+T)=A_{n}(t) e^{-i 2 \pi \alpha}
$$

where $T=2 \pi / \Delta$. The problem then reduces to determining $A_{n}(t)$ for $0 \leq t \leq T$. For this, we introduce the function

$$
B(x ; \alpha)=\sum_{m \in \mathbb{Z}} \frac{e^{-i m x}}{m+\alpha},
$$

for $\alpha \notin \mathbb{Z}$ and $0<x<2 \pi$. In terms of $B$, we have

$$
\begin{aligned}
& A_{n \neq 0}(t)=|C|^{2} e^{-i \alpha \Delta t} \frac{e^{-i n \Delta t}-1}{n} B(\Delta t ; \alpha), \\
& A_{0}(t)=-|C|^{2} e^{-i \alpha \Delta t} \frac{\partial}{\partial \alpha} B(\Delta t ; \alpha) .
\end{aligned}
$$

Now by considering the Fourier series of $e^{i \alpha x}, 0 \leq x \leq 2 \pi$, we have

$$
B(x ; \alpha)=\frac{2 \pi i}{e^{i 2 \pi \alpha}-1} e^{i \alpha x}, \quad 0<x<2 \pi .
$$



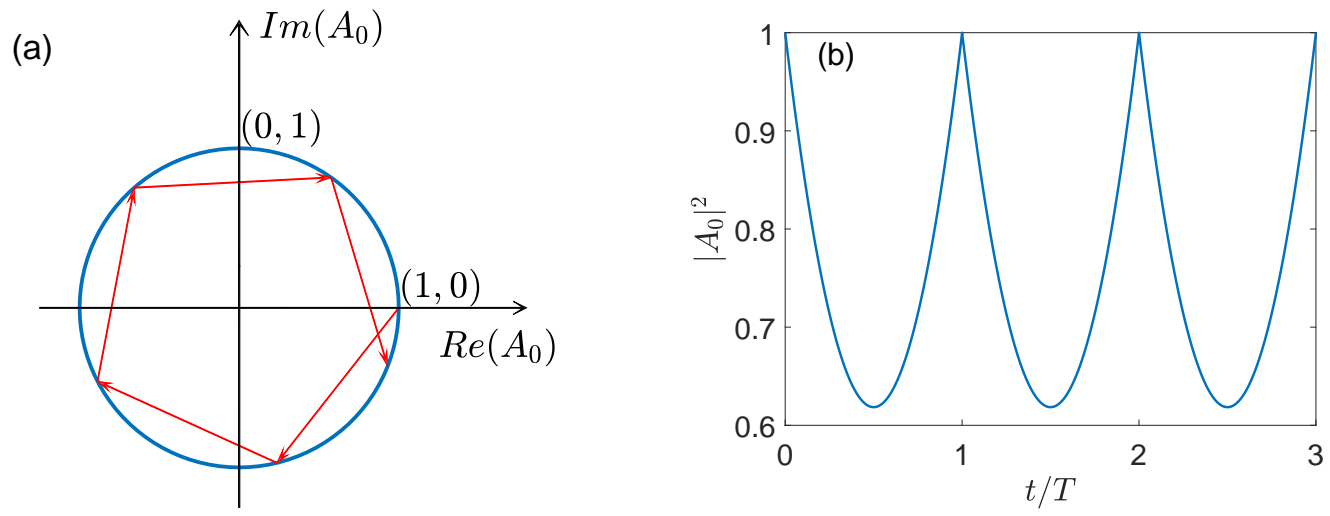

Figure 1. (Color online) (a) Trajectory of the survival amplitude $A_{0}$ in the complex plane; (b) Time evolution of the survival probability $\left|A_{0}\right|^{2}$. The parameters are $\Delta=1$, $g=0.25$.

Substituting this explicit expression into (22) and (23), after some straightforward calculation, we get

$$
\begin{aligned}
& A_{n \neq 0}(t)=\frac{g}{n(\Delta+i \pi g)}\left(e^{-i n \Delta t}-1\right), \\
& A_{0}(t) \quad=1-\frac{i g \Delta t}{\Delta+i \pi g}
\end{aligned}
$$

for $0 \leq t \leq T$. Their values at an arbitrary time can then be determined by using (20).

We note that $A_{0}(t)$ is a linear function of time $t$ when $0 \leq t \leq T$. In this interval, it interpolates linearly between the end values $A_{0}(t=0)=1$ and $A_{0}(t=$ $T)=(\Delta-i \pi g) /(\Delta+i \pi g)=e^{-i 2 \pi \alpha}$, whose absolute values are both unity. This means that after time $T$, the system revives completely - The probability initially flew to other levels has all flown back. Indeed, by (25), $A_{n \neq 0}(t=T)=0$. Apparently, like that in a harmonic oscillator, the perfect revival here is a consequence of the equidistant spectrum of the model.

Together with (20), the fact that $A_{0}$ interpolates linearly between 1 and $e^{-i 2 \pi \alpha}$ in the first period of $[0, T]$ means that it will do so between $e^{-i 2 \pi \alpha}$ and $e^{-i 4 \pi \alpha}$ in the second period of $[T, 2 T]$, and so on. Overall, the picture is that $A_{0}$ follows a trajectory in the complex plane like a ball does in a circular billiard. See figure 1(a) for illustration.

The survival probability $\left|A_{0}\right|^{2}$ is then a periodic function of time which returns to unity when $t$ is an integral multiplier of $T$. In the first period, it has the explicit expression of

$$
\left|A_{0}(t)\right|^{2}=1-\frac{g^{2} \Delta^{2}}{\Delta^{2}+\pi^{2} g^{2}} t(T-t)
$$

which is a polynomial of order two. The curve of $\left|A_{0}\right|^{2}$ is illustrated in figure 1(b). Note that whenever it returns to unity, it shows a cusp.

With (25), (26), and (27), we have reproduced the main results of [5]. Technically, the derivation here is not necessarily simpler; but conceptually, it might be more natural, as it is based on the spectral decomposition of the time evolution operator $e^{-i H t}$, which is 
a standard way to evolve a wave function. It also brings more insight into the problemWe now understand why $A_{0}$ is a nonsmooth function of $t$, which is the main point of [5] - The coefficients of its Fourier series (19) just decay too slowly [18].

$$
\begin{aligned}
& H=-\sum_{m=-\infty}^{\infty}\left(a_{m}^{\dagger} a_{m+1}+a_{m+1}^{\dagger} a_{m}+\frac{U}{2} a_{m}^{\dagger} a_{m}^{\dagger} a_{m} a_{m}\right)+V a_{0}^{\dagger} a_{0} \\
& Z=\sum_{\text {odd-parity }} e^{-\beta E_{n}}+\sum_{\text {even-parity }} e^{-\beta E_{n}}=\int d E\left(\rho_{\text {odd }}(E)+\rho_{\text {even }}(E)\right) e^{-\beta E}
\end{aligned}
$$

\section{Conclusions and discussions}

In conclusion, we have solved analytically the eigenvalues and eigenstates of a toy model, which arose in some quantum quench dynamics problem. This on the one hand fills a gap, and on the other hand provides a different (possibly more natural) approach to studying its dynamics.

The toy model should be of some pedagogical value, as the model itself and the solution presented here are all simple enough. It can serve as a good exercise in a undergraduate course of quantum mechanics.

\section{Acknowledgments}

The authors are grateful to J. Guo and K. Yang for their helpful comments. This work is supported by the National Science Foundation of China under Grant No. 11704070.

\section{References}

[1] Zakhariev B N and Suzko A A 1990 Direct and Inverse Problems: Potentials in Quantum Scattering (Springer-Verlag, Berlin)

[2] Weber T A and Pursey D L 1995 Extended Gel'fand-Levitan method leading to exactly solvable Schrödinger equations with generalized Bargmann potentials Phys. Rev. A 523923

[3] Zhang J M, Braak D and Kollar M 2012 Bound states in the continuum realized in the onedimensional two-particle Hubbard model with an impurity Phys. Rev. Lett. 109116405

[4] Longhi S and Della Valle G 2013 Tamm-Hubbard surface states in the continuum J. Phys.: Condens. Matter 25235601

[5] Zhang J M and Yang H T 2016 Cusps in the quench dynamics of a Bloch state EPL 11460001

[6] Zhang J M and Yang H T 2016 Sudden jumps and plateaus in the quench dynamics of a Bloch state EPL 11610008

[7] Zhang J M and Liu Y 2018 Dynamical Friedel oscillations of a Fermi sea Phys. Rev. B 97075151

[8] Stey G C and Gibberd R W 1972 Decay of quantum states in some exactly soluble models Physica (Amsterdam) 601

[9] Zhang J M and Liu Y 2016 Fermi's golden rule: its derivation and breakdown by an ideal model Eur. J. Phys. 37065406

[10] Glushkov A V and Ivanov L N 1993 DC strong-field Stark effect: consistent quantum-mechanical approach J. Phys. B: At. Mol. Opt. Phys. 26 L379

[11] Moiseyev N 1998 Quantum theory of resonances: calculating energies, widths and cross-sections by complex scaling Phys. Rep. 302211 
[12] Glushkov A V, Khetselius O Yu and Malinovskaya S V 2008 Spectroscopy of cooperative laserCelectron nuclear effects in multiatomic molecules. Molec. Phys. 1061257

[13] Glushkov A V, Malinovskaya S V, Khetselius O Yu, Loboda A V, Sulharev D and Lovett L 2009 Green's function method in quantum chemistry: New numerical algorithm for the Dirac equation with complex energy and Fermi-model nuclear potential Int. J. Quant. Chem. 1091717

[14] Khetselius O 2012 Spectroscopy of cooperative electron-gamma-nuclear processes in heavy atoms: NEET effect J. Phys.: Conf. Ser. 397012012

[15] Aigner M and Ziegler G M 2013 Proofs from THE BOOK 4th edn (Springer, Berlin)

[16] Ding J and Zhou A 2007 Eigenvalues of rank-one updated matrices with some applications Applied Mathematics Letters 201223

[17] Tricomi F G 1957 Integral Equations (Interscience Publishers, London)

[18] Yang K L and Zhang J M 2018 From the smoothness of the initial state to that of the autocorrelation function arXiv:1811.11338 